\title{
Stereotactic body radiotherapy for oligo-recurrence in the liver in a patient with esophageal carcinoma: A case report
}

\author{
ATSUTO KATANO, HIDEOMI YAMASHITA and KEIICHI NAKAGAWA \\ Department of Radiology, The University of Tokyo Hospital, Tokyo 113-8655, Japan
}

Received June 27, 2017; Accepted September 28, 2017

DOI: $10.3892 / \mathrm{mco} .2017 .1441$

\begin{abstract}
Stereotactic body radiation therapy (SBRT) is a safe and effective treatment for solitary cancerous lesions. The present study reported a rare case of sustained remission after SBRT for oligorecurrence in the liver in a patient with esophageal carcinoma. A 60-year-old Japanese man presented with a chief complaint of dysphagia. On medical examination, the patient was diagnosed as having squamous cell carcinoma of the esophagus that was clinically staged as T4bN1M0. The patient received definitive concurrent chemoradiotherapy, consisting of four 28-day cycles of chemotherapy comprising nedaplatin $80 \mathrm{mg} / \mathrm{m}^{2}$ on day 1 and S-1 $120 \mathrm{mg} /$ body orally on days $1-14$, with radiotherapy comprising a total of $50 \mathrm{~Gy}$ in daily fractions of 2 Gy. After a 9-month disease-free interval following the primary treatment, a solitary liver metastasis was identified. The patient underwent SBRT using a radiation dose of 48 Gy in 4 fractions and two 28-day cycles of adjuvant chemotherapy comprising nedaplatin $80 \mathrm{mg} / \mathrm{m}^{2}$ on day 1 , and S-1 $100 \mathrm{mg} /$ body orally on days $1-14$. The patient exhibited no signs of recurrence for 3 years with sustained local control. SBRT may be considered a treatment option for patients with relapsed esophageal cancer with oligo-recurrence in the liver.
\end{abstract}

\section{Introduction}

Esophageal carcinoma is a life-threatening disease. Despite adequate initial treatment, patients remain at high risk of recurrence. Even after esophagectomy following neoadjuvant chemoradiotherapy, $\sim 30 \%$ of patients develop recurrent disease (1). Recurrences predominantly occur in the regional lymph nodes or in distant organs, including the lung, liver, bone, brain, kidney, adrenal glands and skin (2). The liver is the most frequent site of distant recurrence $(3,4)$.

Correspondence to: Dr Hideomi Yamashita, Department of Radiology, The University of Tokyo Hospital, 7-3-1 Hongo, Bunkyo-ku, Tokyo 113-8655, Japan

E-mail: yamachan07291973@yahoo.co.jp

Key words: esophageal cancer, liver metastasis, stereotactic body radiotherapy, oligo-recurrence
The role of surgical resection in the treatment of liver metastases from esophageal carcinoma is controversial. Complete surgical resection is a well-accepted treatment for metastatic liver disease from colorectal cancer, with 5-year survival rates of $25-40 \%$ (5). Huddy et al reported on four patients who underwent successful resection of recurrent liver metastases from esophageal cancer (6). Cryosurgical ablation, transarterial embolization, and radiofrequency ablation also remain as options for management of liver metastases in patients who are unsuitable for surgery.

Stereotactic body radiation therapy (SBRT) can also be considered an effective, safe, and non-invasive therapeutic option for unresectable liver metastases (7). We selected SBRT to treat oligo-recurrence in the liver in a patient with relapsed esophageal cancer, who had a remarkable clinical response without severe adverse events.

\section{Case report}

A 60-year-old Japanese man presented with a chief complaint of dysphagia in December 2012. His alcohol consumption was $60 \mathrm{~g} /$ day and he had smoked 20 cigarettes per day for the previous 40 years. Gastrointestinal fiberscopy revealed a Borrmann type II lesion invading the full circumference of the esophagus at $21-28 \mathrm{~cm}$ from the incisor teeth. The mucosa was biopsied and the diagnosis was moderately to poorly differentiated squamous cell carcinoma. A computed tomography (CT) scan showed a thickened upper-middle thoracic esophageal wall that was broadly attached to the membranous wall of the trachea and an absence of the fat plane, indicating tumor invasion. The CT scan also showed enlarged right upper thoracic paraesophageal and left paratracheal lymph nodes. The patient was staged clinically as T4bN1M0 according to the 7th edition of the American Joint Committee on Cancer TNM staging system.

The patient received definitive concurrent chemoradiotherapy consisting of four 28-day cycles of chemotherapy (nedaplatin $80 \mathrm{mg} / \mathrm{m}^{2}$ on day 1 and S-1 $120 \mathrm{mg} /$ body orally on days 1-14) and radiotherapy (four-field oblique box, parallel opposed pair, $10 \mathrm{MV}$ X-ray beams; $50 \mathrm{~Gy}$ in daily fractions of 2 Gy). He experienced acute adverse events, including grade 2 fatigue, grade 2 esophagitis, grade 1 anemia, grade 3 leukopenia, and grade 3 thrombocytopenia (Common Terminology Criteria for Adverse Events version 4.0). After the chemoradiotherapy, a CT scan showed improvement of 
the thickened esophageal wall and that the swollen lymph nodes had markedly reduced in volume. Gastrointestinal fiberscopy revealed no obvious abnormalities, except for a scar-like lesion at the site of the primary lesion in the esophagus. Accordingly, this treatment was deemed to have resulted in a complete response. Follow-up investigations included a physical examination, assessment of laboratory data, and a CT scan.

In December 2013, an abdominal CT scan revealed a solitary nodule measuring $18 \mathrm{~mm}$ in diameter in segment 8 of the liver (Fig. 1) that was pathologically confirmed by a needle biopsy to be squamous cell carcinoma and was compatible with metastasis from the esophageal cancer. The patient elected to receive SBRT (Fig. 2) followed by two 28-day cycles of adjuvant combination chemotherapy (nedaplatin $80 \mathrm{mg} / \mathrm{m}^{2}$ on day 1 and S-1 $100 \mathrm{mg} /$ body orally on days 1-14). The SBRT consisted of eleven $6 \mathrm{MV}$ X-ray beams including a non-coplanar beam arrangement and 48 Gy in once-daily fractions of 12 Gy delivered to $95 \%$ of the planning target volume.

A CT scan showed that the nodular lesion in segment 8 of the liver was markedly reduced in volume (Fig. 3). The patient showed no signs of recurrence for $\sim 3$ years. However, in January 2017, he noticed dysphagia and a CT scan revealed a large lesion located on the right lateral wall of the oropharynx and another intrahepatic lesion, which were considered a recurrence of esophageal cancer. He underwent salvage chemotherapy consisting of docetaxel $70 \mathrm{mg} / \mathrm{m}^{2}$ intravenously at 3-week intervals and palliative irradiation (30 Gy in daily fractions of $3 \mathrm{~Gy}$ ) to the oropharynx and the metastatic liver lesion.

\section{Discussion}

Metastatic recurrence is associated with poor clinical outcome in cancer treatment. However, as in our case, there is a preliminary state during which patients with cancer have a limited number of relapsed sites with a controlled primary lesion. The term for this state is 'oligo-recurrence', which was defined by Niibe et al $(8,9)$. It originates from Hellman and Weichselbaum's hypothesis of oligometastases, that is, a clinical disease state in which tumors early in the evolution of metastatic progression produce metastases that are limited in number and location (10). Appropriate local treatment could be efficacious for these 'oligometastatic' lesions. Indeed, Iitaka et al have reported long-term survival in a patient with a recurrence of esophageal cancer that was treated by a multimodal combination of appropriate systemic and local therapy (11).

SBRT is a safe and locally effective treatment for patients with inoperable oligometastases $(12,13)$. A retrospective study of 51 patients with metastatic liver tumors in Japan reported that SBRT was associated with a local control rate of $64.2 \%$ and an overall survival rate of $72 \%$ at 2 years, with no significant (over grade 2) elevation of liver enzymes during treatment (14). These outcomes confirm that SBRT has a beneficial effect in patients who are unsuitable for surgery.

However, the indication for SBRT in the management of oligometastases should be considered carefully. Although the

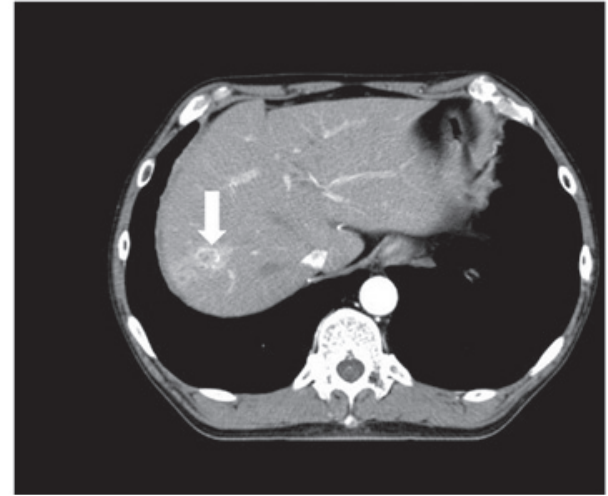

Figure 1. Contrast-enhanced CT scan of the abdomen in December 2013 showing a lesion measuring $18 \mathrm{~mm}$ in segment 8 of the liver (indicated by white arrow). CT, computed tomography.
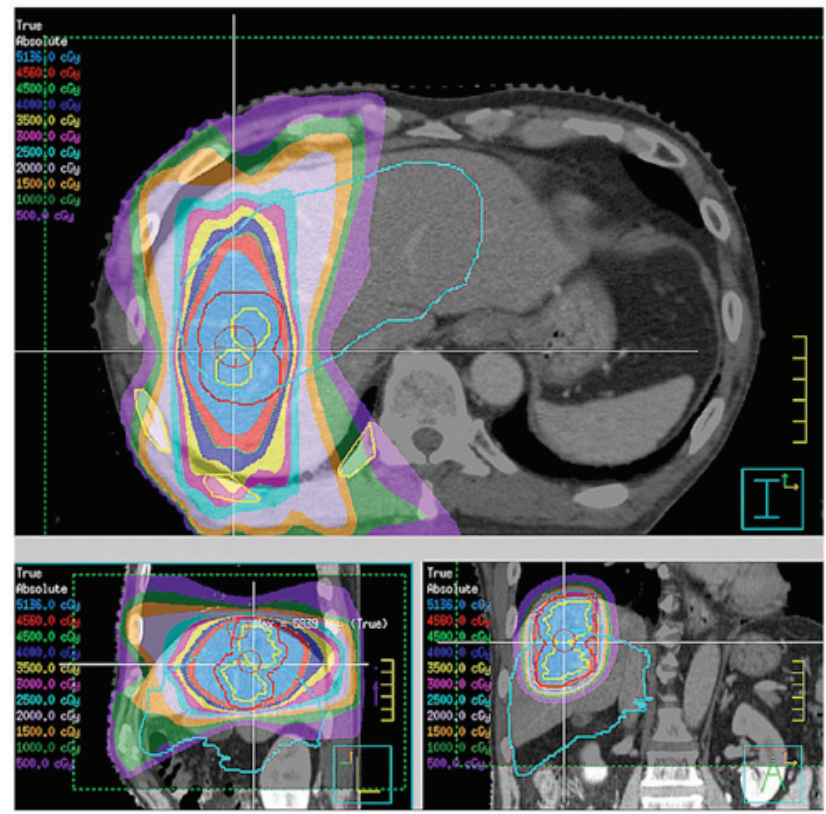

Figure 2. Distribution of the SBRT dose for a liver tumor. The sky-blue line indicates the position of the liver during the inhale phase. The red line indicates the planning target volume. The radiation isodose lines are shown in the upper left corner. The red area indicates the $95 \%$ isodose of the prescribed dose. SBRT, stereotactic body radiation therapy.

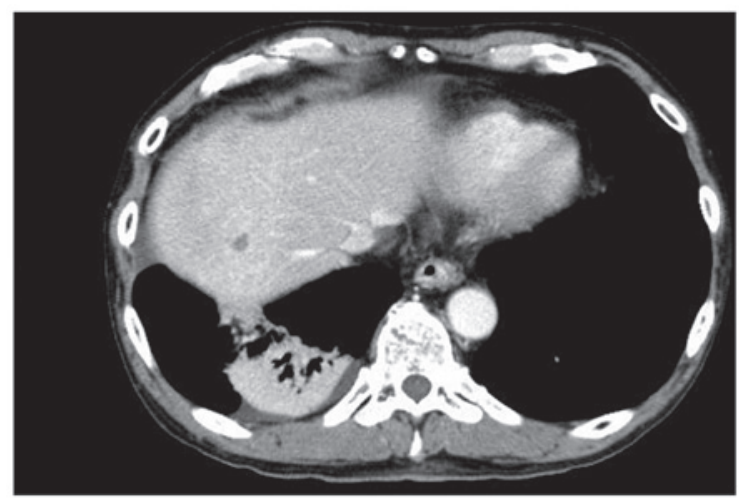

Figure 3. Contrast-enhanced CT scan of the abdomen in September 2014 showing a significant reduction in the size of the hepatic metastasis after SBRT and consolidation in the irradiated field in the posterior segment of the right lower lobe. CT, computed tomography. SBRT, stereotactic body radiation therapy. 
response rate in patients treated by SBRT is high, intrahepatic recurrence has been observed in a proportion of patients. Milano et al reported that $45 \%$ of their patients with initial oligometastases confined to the liver developed local recurrence after SBRT (15).

Appropriate management of an oligometastatic state has a significant role in the management of cancer patients. Egawa et al reported successful control of liver metastasis from esophageal cancer for 1 year following SBRT (16). SBRT might be considered as a good treatment option for liver oligo-recurrence of esophageal carcinoma.

\section{References}

1. Meguid RA, Hooker CM, Taylor JT, Kleinberg LR, Cattaneo SM II, Sussman MS, Yang SC, Heitmiller RF, Forastiere AA and Brock MV: Recurrence after neoadjuvant chemoradiation and surgery for esophageal cancer: Does the pattern of recurrence differ for patients with complete response and those with partial or no response? J Thorac Cardiovasc Surg 138: 1309-1317, 2009.

2. Quint LE, Hepburn LM, Francis IR, Whyte RI and Orringer MB: Incidence and distribution of distant metastases from newly diagnosed esophageal carcinoma. Cancer 76: 1120-1125, 1995.

3. Mariette C, Balon JM, Piessen G, Fabre S, Van Seuningen I and Triboulet JP: Pattern of recurrence following complete resection of esophageal carcinoma and factors predictive of recurrent disease. Cancer 97: 1616-1623, 2003.

4. Hulscher JB, van Sandick JW, Tijssen JG, Obertop H and van Lanschot JJ: The recurrence pattern of esophageal carcinoma after transhiatal resection. J Am Coll Surg 191: 143-148, 2000.

5. Abbas S, Lam V and Hollands M: Ten-year survival after liver resection for colorectal metastases: Systematic review and meta-analysis. ISRN Oncol 2011: 763245, 2011.
6. Huddy JR, Thomas RL, Worthington TR and Karanjia ND: Liver metastases from esophageal carcinoma: Is there a role for surgical resection? Dis Esophagus 28: 483-487, 2015.

7. Scorsetti M, Arcangeli S, Tozzi A, Comito T, Alongi F, Navarria P, Mancosu P, Reggiori G, Fogliata A, Torzilli G, et al: Is stereotactic body radiation therapy an attractive option for unresectable liver metastases? A preliminary report from a phase 2 trial. Int J Radiat Oncol Biol Phys 86: 336-342, 2013.

8. Niibe Y and Hayakawa K: Oligometastases and oligo-recurrence: The new era of cancer therapy. Jpn J Clin Oncol 40: 107-111, 2010.

9. Niibe Y and Chang JY: Novel insights of oligometastases and oligo-recurrence and review of the literature. Pulm Med 2012: 261096, 2012.

10. Hellman S and Weichselbaum RR: Oligometastases. J Clin Oncol 13: 8-10, 1995.

11. Iitaka D, Shiozaki A, Fujiwara H, Ichikawa D, Okamoto K, Komatsu S, Murayama Y, Ikoma H, Kuriu Y, Nakanishi M, et al: Case involving long-term survival after esophageal cancer with liver and lung metastases treated by multidisciplinary therapy: Report of a case. Surg Today 43: 556-561, 2013.

12. Almaghrabi MY, Supiot S, Paris F, Mahé MA and Rio E: Stereotactic body radiation therapy for abdominal oligometastases: A biological and clinical review. Radiat Oncol 7: 126, 2012.

13. de Vin T, Engels B, Gevaert T, Storme G and De Ridder M: Stereotactic radiotherapy for oligometastatic cancer: A prognostic model for survival. Ann Oncol 25: 467-471, 2014.

14. Yamashita H, Onishi H, Matsumoto Y, Murakami N, Matsuo Y, Nomiya $\mathrm{T}$ and Nakagawa K; Japanese Radiological Society multi-institutional SBRT study group (JRS-SBRTSG): Local effect of stereotactic body radiotherapy for primary and metastatic liver tumors in 130 Japanese patients. Radiat Oncol 9: 112, 2014.

15. Milano MT, Katz AW and Okunieff P: Patterns of recurrence after curative-intent radiation for oligometastases confined to one organ. Am J Clin Oncol 33: 157-163, 2010.

16. Egawa T, Okubo Y, Kemmochi T, Mori T, Sato S, Nishiya S, Mihara K, Ito Y, Makino $\mathrm{H}$ and Nagashima A: A case of liver metastasis from esophageal cancer treated with stereotactic body radiation therapy. Gan To Kagaku Ryoho 40: 1850-1852, 2013. (In Japanese). 